\title{
Bepliner ophthalmologische Gesellschaft
}

\section{Sitzung vom 17. April 1902}

Lehmann, Demonstration eines Fades von beiderseitiger totaler Ophthalmoplegia externa bei einem 1 Tjährigen Mädchen mit beiderseitiger Schwäche der Nervi faciales.

liist $\pi$ miente.

63

Fehr: Zur Kenntníss der Netzhautablösung. Dauerheilung (seit 11 Jaliren) einer myopischen Netzbautablüsung, bei welcher vor 11 Jaliren die Scleralpuaction gemacht worden war. Jetzt Ablüsung auf deni andereii Auge, iiu Bereich eines bereits vor 9 Jaliren con-statierten Netzhautrisses.

Stein dorff: Ueber Star operation am Glotzauge. Star-Extraction bei einer 62järigen Frau niit hochgradiger Myopie und Basedow-scherlvranklieit. - Urn die Gefahren des Umklappens der papierdüimen! - Hornhaut uud uni Glaskörpervorfall zu vermeiden, empfiehlt es sicli zweizeitig zu operiren, d. h. eine präparatorische Iridectomie voran-zuscliicken, ausserdem das Einlegen eines Sperrers zu umgelieu, viel-melir die Lider durch einen Assistenten offen halten zu lassen. Rosenste in: Ueber He mi an opsie. Bericht über eine liomonynie rechtsseitige Halbblindlieit, die im Anschluss au Apoplexie auftrat, sicli aber in wenigen Wochen vollständig zurückbildete. May demonstrirt 1. eine Kranke mit besonders gut erhaltener Arteria hyaloidea.

2. Eine Kranke mit beiderseits erhaltener Membrana pupillaris perseverans und markhaitigen Sehnervenfasern.

Пirschberg: Ueber ßehandlung des Keratoconus. H. er-innert daran, dass die Beliandlung dieses L·eidens nacb v. Graefe < larin bestaud. dass die Spitze des Hornhautkegels Yorsiclitig abgetragen und dann mit Argentina geätzt wurde, um durch Narbenzug Abflacbung des Kegels zu erzielen. Späterhin wurde statt des Höllensteines eine schwach glühende Olive benutzt. - II. stellt einen Mann vor, dem er vor 9 und 11 Jaliren die Spitze des Kegels gebrannt und dann tätowirt hatte. Da die \rorwölbung der Hornhaut neuerdings fortschritt, durch Eserin aber die Sehschärfe wesentlich gebessert wurde, so färbte II. um die Pupille lierum eine riugfürmige Zone durch Tittowirung in mehreren Sitzungen, wodurch $S$ von $5 / 50$ auf fast 5/20 anstieg.

Fehr erzielte gute Erfolge in einem Falle von Keratomalaeie durch 7 Wochen lang foitgesetztes feuchtes Verbinden der Augeu.

C. Hamburger.

Instrumente.

Ein kleiner Sterilisator für Vepband und Instpumente zum Gebpauche bei aug ·enäpztliehen Operationen und in dep kleinen

Chipupg $\cdot$ ie.

Von Prof. Dr. W. KOSÏER, Gzn.

in Leiden. (Mit zwci Figuren im Text.)

S. Sclion längst fühlte ich bei der Ausfiihrung von Augenoperationen iiussorhalb des Krankenhauses das Bedürfniss nacli einem Apparate welcher es ermöglicht 
in wenigen Minuten die benöthigten Instrumente 